\title{
Self-Cycling Modularized Smart Grid Application Integration
}

\author{
Y.P. Li, J.B. Xin \\ State Grid Jiangxi Electric Power Research Institute \\ Nanchang, 330096, China
}

\author{
S. Liang, W. Xu \\ Nanrui Technology Co., Ltd \\ Nanjing, 211106, China
}

\begin{abstract}
The essence of the construction of smart grid demonstration project is an integration process of various types of technology, system and device. With the integration of various kinds of adjustable resources including distributed power supply, reactive power compensation devices, and energy storage devices etc, the traditional passive distribution network is evolving into active distribution network, and low carbon power grid comprehensive demonstration project is a composition of distributed generation, energy storage, active load and the associated control equipment. On the basis of establishing unified information platform including various sub modules, it is significantly important to achieve low carbon optimized dispatch through the coordination of the modules.
\end{abstract}

Keywords-modularity; smart grid; low carbon

\section{INTRODUCTION}

With the continuous increase of the global resource environment pressure, social requirements on environmental protection, energy saving and emission reduction and sustainable development is being required greatly; at the same time, the continuous development of electricity market and users requirement for electricity quality and reliability decides that the electric networks of the future must be able to provide more safe, reliable, clean and high quality power supply, to adapt to the needs of various types of energy power generation mode, and to adapt to the need of highly market-oriented power transactions.

A smart grid is the use of sensors, communications, computational ability and control to enhance the overall functionality of the electric power delivery system. This permits several functions which allow optimization in combination of the use of bulk generation and storage, transmission, distribution, distributed resources and consumer end uses toward goals which ensure reliability and optimize the use of energy, mitigate environmental impact, manage assets, and contain cost.

Low carbon power grid comprehensive demonstration project is a composition of distributed generation, energy storage, active load and the associated control equipment [1-4] On the basis of establishing an unified information platform including various sub module, it is significantly important to achieve low carbon optimized dispatch through the coordination of the modules [5-6].

\section{UNIFIED INFORMATION PLATFORM}

Gongqing city is located in the north of Jiangxi province,
China, the middle of the Nanchang-Jiujiang industrial corridor. The existing area of Gongqing is $170 \mathrm{~km} 2$, with the population of 100 thousands. In June 2011, the Japanese NEDO launched the Gongqing "smart community" demonstration project with Jiangxi Province officially in Tokyo. The demonstration project has been constructed by Toshiba, Itochu, and State Grid of China Corporation etc, and is expected to be complete in 2014. The core idea of the project is to construct an intelligent technology demonstration area by considering both economic and low carbon development.

Based on the already constructed integrated visualization platform for the Jiangxi Gongqing city smart grid, we researched and developed an unified information support platform including hardware, software, historical database and middle ware data bus etc, to provide support for the six professional application modules of distribution network dispatch, operation and maintenance, marketing, planning, distributed power supply and low carbon evaluation and validation.

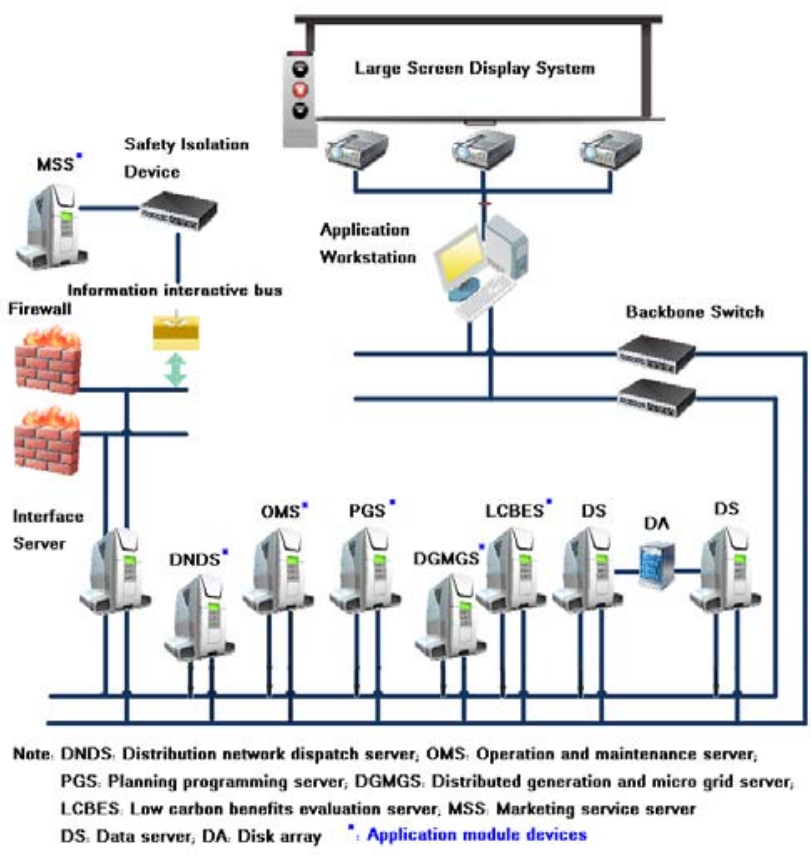

FIGURE I. THE UNIFIED INFORMATION PLATFORM: HARDWARE STRUCTURE.

The illustration of the unified information platform 
hardware structure is shown in Figure 1: the two data servers and the disk array to form a local data center, the six application servers to demonstrate the visualization data of six professional application modules and finish the WEB page login. And the software structure of the platform is illustrated in Figure 2.

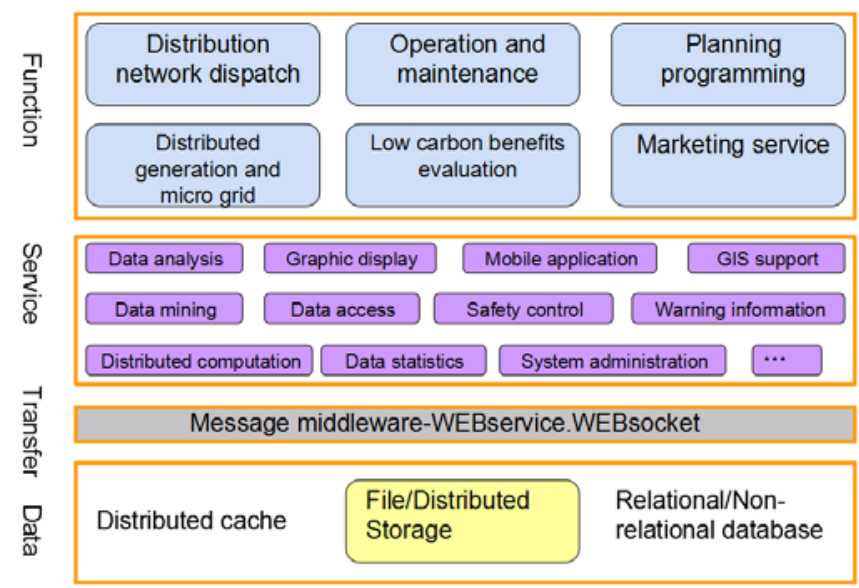

FIGURE II. THE UNIFIED INFORMATION PLATFORM: SOFTWARE STRUCTURE.

By utilizing the IEB information interactive bus, the platform can realize the data exchange, data sharing and application integration with the currently existed information systems including production management system, GIS system, marketing management system, 95598 system, the electric energy data acquisition system, dispatching automation system, power quality monitoring system, power voltage monitoring system, WSN device status monitoring, electric vehicle charging/exchange station, and intelligent residential community master station system etc.

\section{SELF-CYCLING APPLICATION INTEGRATION}

To abbreviate, we use DNDM for distribution network dispatch module, OMM for operation and maintenance module, PGM for planning programming module, DGMGM: distributed generation and micro grid module, LCBEM: low carbon benefits evaluation module, and MSM for marketing service module.

Based on the current power grid operation state, in order to reduce the network loss as the constraint conditions and improve the efficiency and safety of power grid operation as the objective, the DNDM module utilize the power grid real-time data to establish the data analysis model, make optimization scheduling strategy of regional power grid operation, photovoltaic power generation system, the electric vehicle charging and discharging system and storage system. On this basis, online simulation is performed to generate simulation data that meets operating requirements, including three phase power flow, network loss and power. Then the simulation data was provided to both the OMM and LCBEM modules.

After that, the OMM module performs the power grid operation risk assessment, including the comparison of the operation status of switch, bus and load with the ideal model, and submit the evaluation results to the DNDM module. At the same time, the OMM module performs the online risk evaluation of all the transformers, lines and load etc in the whole area, then the evaluation results is provided to the PGM module.

When the DGMGM module received scheduling orders from the DNDM module, it makes the corresponding adjustment of photovoltaic power generation, energy storage devices and micro grid operation state, and then the operation condition information is passed back to the DNDM module.

After the MSM module received scheduling orders from the DNDM module, it makes the corresponding adjustment of the charging and discharging strategy for electric vehicles, orderly charging control of electric vehicles, and then the operation condition information is passed back to the DNDM module.

Based on the current grid operation state, the LCBEM module computes both carbon emission index and real-time line loss, also, this computation was performed on the simulation data received from the DNDM module, then the results of both carbon and loss reduction was formed. The above computation was done again after receiving the power grid operation data with optimal adjustment. In this way, the effectiveness of both the multi-energy complementary low carbon optimization scheduling and the carbon reduction was verified.

\section{CONCLUSION}

We developed an unified information support platform including hardware, software, historical database and middle ware data bus etc, to provide support for the six professional application modules of distribution network dispatch, operation and maintenance, marketing, planning, distributed power supply and low carbon evaluation and validation. On the basis of the platform, both the multi-energy complementary low carbon optimization scheduling and the carbon reduction can be realized through the coordination of six professional application modules.

\section{REFERENCES}

[1] Kang Chongqing, Chen Qixin, et al. Prospects of low-carbon electricity[J]. Power System Technology, 2009, 33(2): 1-7.

[2] Lu Siyu, Lou Suhua, et al. A model for generation expansion planning of power system based on carbon emission trajectory model under low-carbon economy[J]. Transactions of China Electrotechnical Society, 2011, 26(11): 175-181.

[3] Hondo H. Life cycle GHG emission analysis of power generation systems: Japanese case[J]. Energy, 2005(30): 2042-2056.

[4] Zhou Tianrui, Kang Chongqing, et al. Analysis on distribution characteristics and mechanisms of carbon emission flow in electric power network[J]. Automation of Electric Power Systems, 2012, 36(15): 39-44.

[5] Xiao Xiangning, Chen Zheng, et al. Integrated mode and key issues of renewable energy sources and electric vehicles' charging and discharging facilities in microgrid[J]. Transactions of China Electrotechnical Society, 2013, 28(2): 1-14.

[6] Li Da-yong, Ma Dongxue, et al. Application of network information visualization[J]. Power System Protection and Control, 2009, 37(23): 156-158. 